\title{
Berliner Medizinische Gesellschaft
}

\section{Sitzung vom 21. X. 1925}

Vor der Tagesordnung: C. Hamburger, Glaukombckaiidlung (lurch Einträufelung cines konzentrierten Suprareningemisches (SR).

Das ursprünglich gegen Glaukom angewandte SR (linksdrehenã) hat Hamburger vor einem halben Jahr durch ein røcAfedrehendes Arznei-gemisch (,, Glaukosan”) ersetzt1), weil dieses ohne Einfluß auf den allgemeinen Blutdruck ist, mithin auch bei ältesten Leuten unbesorgt subkonjunktival injiziert werden kann. Da aber diese Form der Appli-kation unbequem ist und auf die Dauer lästig wird, so hat H. die Injek-tion durch eine Einträufelung zu ersetzen gesucht. Zum Beweise, daß dies gelungen, stellt er sechs Patienten mit Glaucoma simplex vor, bis 80 Jahre alt. Um die Einträufelung wirksam zu machen, war starke Er-höhung der Konzentration und Rückkehr zu den Lf«Ãs-Salzen nötig. Die Besorgnis, die Einträufelung könnte den Blutdruck stark steigern, erwies sich als unbegründet (bei sechs Patienten wurde nach RivaRocci kontrolliert): und doch dringt die Gefäßkonstriktion so tief, daß - häu-fig - weiße anämische Flecke in der Gesichishaiit entstehen, oft bis zu den Mundwinkeln herab (subkonj. Injektion ist streng kontraindiziert, sie führt im Tierversuch den Tod herbei; aufgetropjt hingegen ist das Medikament quoad vitam harmlos). - Auch für die Hornhaut bringen die in Frage kommenden Lösungen keine ernsthafte Gefahr. (Im Tierversuch benutzte H. die Linkssaize bis zuı\%(!), die Rechtssalze biszu 33\%(!)·) Beim Menschen kommen weit schwächere Konzentrationen in Anwendung, zusammen mit der optisch inaktiven letzten Vorstufe. Vier mitprüfende Herren sahen gar keine Hornhautveränderung; H. sah aber ganz sicher in einigen

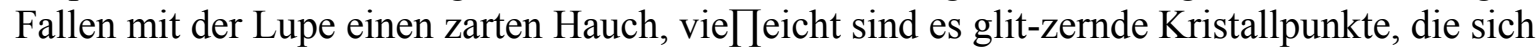
aber stets spurlos in einigen Stunden auflösten; er gibt das Mittel auch den Einäugigen, wovon er zwei vor-stellt, auch den vergeblich Operierten; auch seinem 20jährigen Sohn hat er es, sechsmal, ins gesunde Auge eingetropft; ebenso der Oberin seiner Klinik; es ist auch geeignet als Mydriatikum bei alten Leuten, da es die Pupille bei Hypotonie erweitert. - Das Medikament heißt Glaukosan-Trop†en oder Links-Glaukosan2), weil es die optische Achse links dreht; es kommt (mit den Substanzen der Höchster Farbwerke) von der $\Lambda$.-G. Wölm, Spangenbergbei Casselin Verkehr, in kleinenabgeteilten Quan-

!) Vgl. Med. Klinik 1925, Nr. 40, Ersatzpräparàte für Adrenalin und ihre Bedeutung für die Glaukombehandlung.

2) Packung und Ampullenform schließen jede Verwechslung mit einer Injektion völligaus; zur weiteren Sicherheit dient schon der Name (Glaukosan-Tropfen). Oieiibliche Art des Träufelns genügt freilich nicht; vielmehr ist für den Erfolg entscheidend eine besonäere Technik; sie ist, ebenso wie die Konzentration, Handhabung der ,,TropfampulIe” usw. im Prospekt, welcher der Packung beiliegt, genau beschrieben.

186 Berliner Medizinische Gesellschaft. titäten, denn man braucht zu jeder Sitzung nur einige Tropfen (wirksam fürmehrere Tage); einmal geöffnet und der Luft ausgesetzt, sind stärkereSR-Lösungen leicht zersetzlich. - In historischer Hinsicht erinnert H.daran, daß kaum eine Substanz sich so schwer einführen ließ wie 
-Atropin (!); die Belegstellen werden mitgeteilt1); dem SR mißt er einegroße Zukunft bei, theoretisch wie praktisch. Es sei wichtig, daß manz. B. bei Eserin-Idiosynkrasie den gleichen Effekt mit diesem Mydriati-kum erreiche; übrigens sei nach dessen Anwendung das Miotikum oftwieder wirksam. - Bemerkenswert erscheint, daß bei manchen, nament-lich jüngeren Menschen die Akkommodation völlig ungestört bleibt; beianderen wird sie, vorübergehend, um i-2 D herabgesetzt. - Bisweilenempfiehlt es sich, acht Tage lang Eserin, die nächsten acht Tage Links-glaukom einzuträufeln, also abwechsclnd mit enger und mit weiterPupille zu behandeln. Die Patienten, namentlich die sensibleren, em-pfinden dies Mittel, dem Eserin und Pilocarpin gegenüber, wohltuencl, weil die Miotica allzuviel Licht fortnehmen.2) Selbstbericht. Diagnose und Therapie.

Blaauw, F.E., Syphilom als Komplikation nach einer Staroperatiou. American. Journal of Ophthalm. Bd. 8, Nr. 1, 1925. S. 59/60.

Ein 65jähriger Mann war an einem Auge vor vier Jahren wegenAltersstar operiert. Das Auge war aber, anscheinend nach Infektion, erblindet. Am zweiten Auge wurde derselbe Eingriff mtnmehr mit be-sonderer Vorsicht ausgeführt. Die Operation verlief glatt; der Heil-verlauf in den ersten 10 Tagen war ungestört. Am 14. Tage wurde aufder Iris, im Kammerwinkel unten, also an der dem Operationsschnittenígegengesetzten S'eite, ein gelbliches Gebilde sichtbar, das auch etwasspeckig erschien (Größe 4X2 mm). Ein leichter Entzündungszustanddes Auges lag außerdem vor. Infektion wurde nicht angenommen.Die Wa.-Reaktion war negativ. Auf anitluetische Behandlung hin gingdas Gebilde schnell zurück. Es war 10 Tage nach der ersten und zweiTage nach der zweiten Hg.-Injektion verschwunden. (Jodkali innerlichwar außerdem gegeben.) Unzweifelhaft hatte es sich um ein Syphilomgehandelt. Verf. sah etwas ähnliches schon einmal bei einer 45jährigenFrau. Ein gewisser Zusammenhang mit dem durch den operativenEingriff gesetzten Trauma wird angenommen. Verf. erwähnt, daß erin der Fachliteratur seines Landes, aber auch in der deutschen Literaturkeinen Bericht über eine derartige Beobachtung von anderer Seite fand, auch nicht in dem bezüglichen Abschnitt der Neuauflage des Hand-buches von Graeie-Saemisch (1923). Junius (Bonn).

Cedercveutz, Axel (Vorstand der Univ.-Klinik für Hautkrankheiten in Helsingfors): Siod innersekretorische Störungen auî kongcuital-Iuetischer $\mathrm{C}^{1} / 8$ undlage als Ursache der Hutehinsonschen Trias auîzufassen? (Munch.

Med. Wochenschrift 1925, Nr. 46, S. i960.

Verf. hat in 1913 die Vermutung ausgesprochen, daß Zahnanomalie, Keratitis parenchymatosa und Labyrintherkrankung des Ohres, der Hut-

x. x) Vgl. Hirschberg

Gesch. d. Aug. 1911

S. 10 u. 12; ferner Himly

Krhtn. d. Auges

Berlin 1843

Teil 2

S. 115.

2) Hier folgte die Demonstration einiger Kurven. 